\title{
Does the impact of a plant-based diet during pregnancy on birthweight differ by ethnicity?
}

\author{
M.A. Zulyniak ${ }^{1,2}$ R.J. de Souza $^{3}$, M. Shaikh ${ }^{3}$, D. Desai ${ }^{1,4}$, D.L. Lefebvre ${ }^{1}$, M. Gupta ${ }^{1,5}$, \\ J. Wilson ${ }^{6}$, G. Wahi ${ }^{3,7}$, P. Subbarao ${ }^{8}$, A.B. Becker ${ }^{9}$, P. Mandhane ${ }^{10}$, S.E. Turvey ${ }^{11}$, J. Beyene ${ }^{3}$, \\ S. Atkinson ${ }^{7}$, K. Morrison ${ }^{7}$, S. McDonald ${ }^{3}$, K.K. Teo ${ }^{1,4}$, M.R. Sears ${ }^{1}$ and S.S. Anand ${ }^{1,3}$ \\ ${ }^{1}$ Department of Medicine, McMaster University, Canada, ${ }^{2}$ School of Food Science and Nutrition, University of Leeds, \\ LS17 5BL, ${ }^{3}$ Department of Health Research Methods, Evidence, and Impact, McMaster University, Canada, \\ ${ }^{4}$ Population Health Research Institute, Hamilton Health Sciences and McMaster University, Canada, ${ }^{5}$ Canadian \\ Cardiovascular Research Network, Canada, ${ }^{6}$ Six Nations Health Services, Canada, ${ }^{7}$ Department of Paediatrics, \\ McMaster University, Canada, ${ }^{8}$ Hospital for Sick Children \& Department of Paediatrics, University of Toronto, \\ Canada, ${ }^{9}$ Department of Immunology, Faculty of Medicine, University of Manitoba, Canada, ${ }^{10}$ Department of \\ Pediatrics, Faculty of Medicine and Dentistry, University of Alberta, Canada and ${ }^{11}$ BC Children's Hospital, \\ Department of Paediatrics, Faculty of Medicine, University of British Columbia, Canada.
}

Birthweight is an indicator of newborn health ${ }^{(1)}$ and a strong predictor of health outcomes in later life, including cardiovascular disease, diabetes, and obesity ${ }^{(2)}$. Significant variation in dietary intake during pregnancy between ethnic groups ${ }^{(3)}$ provides an ideal opportunity to investigate the influence of maternal diet on birthweight. We aimed to investigate the impact of maternal dietary patterns on birthweight in four multi-ethnic birth cohorts in Canada.

We analyzed 3,997 full-term mother-infant pairs from diverse ethnic groups. Multivariable regression was used to test the association between 3 principal component analysis-derived diet patterns (plant-based, Western, health-conscious) and birthweight. The foods comprising significant diet patterns were investigated to identify key foods contributing to this association.

No associations were identified between the Western and health-conscious diet patterns and birthweight; however, the plant-based dietary pattern was inversely associated with birthweight $(\beta=-67.6 \mathrm{~g}$ per 1 -unit increase; $\mathrm{P}<0.001)$ and an interaction with nonwhite ethnicity and birthweight was present. Ethnically stratified analyses demonstrates that among white Europeans, maternal consumption of a plant-based diet associated with lower birthweight $(\beta=-65.9 \mathrm{~g}$ per 1 -unit increase; $\mathrm{P}<0.001)$, increased risk of small for gestational age $(\mathrm{SGA} ; \mathrm{OR}=1.46 ; 95 \% \mathrm{CI}: 1.08-1 \cdot 54 ; \mathrm{P}=0.005)$, and reduced risk of large for gestational age $(\mathrm{LGA}$; OR = 0.71; $95 \%$ CI: 0.53-0.95; P = 0.02). Among South Asians, maternal consumption of a plant-based diet associated with a higher birthweight $(\beta=+40 \cdot 5 \mathrm{~g}$ per 1 -unit increase; $\mathrm{P}=0 \cdot 01)$, partially driven by cooked vegetable consumption.

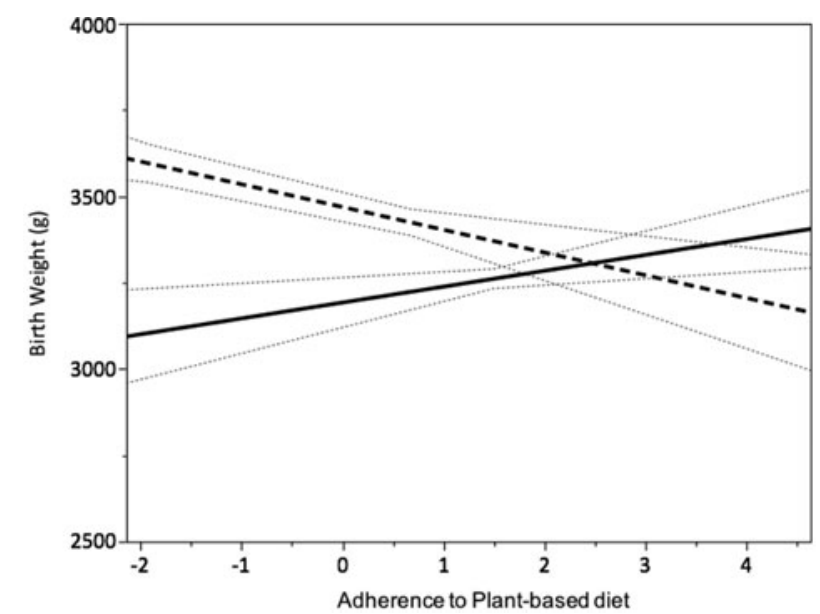

Fig. 1. Multivariable regression between maternal adherence to a plant-based diet (higher score reflects greater adherence) and birthweight in white Europeans (dashed line; $n=2,367$ ) and South Asians (solid line; $n=884$ ). Dotted line is the $95 \%$ confidence interval.

In conclusion, maternal consumption of a plant-based diet during pregnancy is associated with birthweight. Among white Europeans, a plant-based diet is associated with lower birthweight, reduced odds of an infant born LGA, and increased odds of SGA, whereas among South Asians living in Canada, a plant-based diet is associated with increased birthweight.

1. Mikolajczyk RT, Zhang J, Betran AP, et al. (2011) Lancet 377, 1855-1861.

2. Nordman H, Voutilainen R, Laitinen T, et al. (2015) Horm Res Paediatr 85, 11-17.

3. de Souza RJ, Zulyniak MA, Desai D, et al. (2016) J Nutr 146, 2343-2350. 